\title{
FERTILIDADE DO SOLO SOB DIFERENTES USOS AGROFLORESTAIS NA REGIÃO CENTRAL DE RONDÔNIA, BRASIL
}

Soil fertility in different agroforestry uses in the central region of Rondonia, Brazil Desafios

Fertilidad del suelo en diferentes usos agroforestales en la región central de Rondonia, Brasil

Fernando Luíz de Oliveira Corrêa ${ }^{* 1}$, Caio Márcio Vasconcellos Cordeiro de Almeida ${ }^{2}$, Manfred Willy Müller ${ }^{3}$, Adriano Reis Prazeres Mascarenhas ${ }^{4}$

${ }^{1}$ Comissão Executiva do Plano da Lavoura Cacaueira, Superintendência de Desenvolvimento da Região Cacaueira do Estado de Rondônia, Ouro Preto do Oeste, RO, Brasil.

${ }^{2}$ Comissão Executiva do Plano da Lavoura Cacaueira, Superintendência de Desenvolvimento da Região Cacaueira do Estado de Rondônia, Porto Velho, RO, Brasil.

${ }^{3}$ Comissão Executiva do Plano da Lavoura Cacaueira, Diretoria Geral, Brasília, DF, Brasil.

${ }^{4}$ Universidade Federal de Rondônia, Departamento Acadêmico de Engenharia Florestal, Rolim de Moura, RO,

Brasil.

*Correspondência: Estação Experimental da CEPLAC-RO, BR 364, Km 2,5, Ouro Preto do Oeste, RO, Brasil.

CEP: 76.920-000.e-mail fernando.luiz@agricultura.gov.br.

Artigo recebido em 30/06/2019 aprovado em 29/10/2019 publicado em 04/12/2019.

\section{RESUMO}

A implementação de sistemas agroflorestais na amazônia pode ser uma alternativa aos modelos de uso e ocupação do solo tradicionais, pois são baseados em cultivos que favorecem a ciclagem de nutrientes, a partir da associação dos extratos aéreos e subterrâneos entre espécies florestais e agrícolas. Sendo assim, objetivou-se avaliar as condições químicas do solo sob diferentes usos agroflorestais na região central de Rondônia, Brasil. Para isso, adotou-se o delineamento experimental em blocos casualizados, com três repetições e três tratamentos, representados por diferentes formas de cobertura vegetal, sendo eles: T1 - Sistema agroflorestal composto com cacaueiros e fruteiras perenes; T2 - Trilhamento na capoeira com essências florestais; T3 - Capoeira enriquecida com essências florestais e T4 - Floresta primária. De forma geral, os teores de cálcio e o alumínio foram os que mais variaram nos diferentes tipos de cobertura e profundidades do solo. Já em relação as propriedades referentes ao $\mathrm{pH}$ e teores de fósforo e matéria orgânica, não foram observadas diferenças entre a floresta primária e os demais tipos de cobertura.

Palavras-chave: Agrossilvicultura, manejo do solo, área degradada.

\section{ABSTRACT}

The implementation of agroforestry systems in the Amazon can be an alternative to traditional land use and occupation models, as they are based on crops that favor nutrient cycling, based on the association of aerial and underground extracts between forest and agricultural species. Thus, the objective was to evaluate soil chemical conditions under different agroforestry uses in the central region of Rondonia, Brazil. For this, the experimental design was randomized blocks with three replications and three treatments, represented by different forms of vegetation cover, as follows: T1 - Agroforestry system composed of cacao and perennial fruit; T2 - Capoeira trail 
with forest essences; T3 - Capoeira enriched with forest essences and T4 - Primary forest. In general, calcium and aluminum contents varied the most in different types of cover and soil depth. Regarding the properties related to $\mathrm{pH}$ and contents of phosphorus and organic matter, no differences were observed between the primary forest and the other types of cover.

Keywords: Agroforestry, soil management, degraded areas.

\section{RESUMEN}

La implementación de sistemas agroforestales en la Amazonía puede ser una alternativa a los modelos tradicionales de uso y ocupación de la tierra, ya que se basan en cultivos que favorecen el ciclo de nutrientes, basados en la asociación de extractos aéreos y subterráneos entre especies forestales y agrícolas. Por lo tanto, el objetivo era evaluar las condiciones químicas del suelo bajo diferentes usos agroforestales en la región central de Rondonia, Brasil. Para esto, el diseño experimental se realizó en bloques al azar con tres repeticiones y tres tratamientos, representados por diferentes formas de cubierta vegetal, de la siguiente manera: T1 - Sistema agroforestal compuesto por cacao y frutos perennes; T2 - sendero de capoeira con esencias forestales; T3 Capoeira enriquecida con esencias forestales y T4 - Bosque primario. En general, el contenido de calcio y aluminio varió más en diferentes tipos de cobertura y profundidad del suelo. Con respecto a las propiedades relacionadas con el pH y el contenido de fósforo y materia orgánica, no se observaron diferencias entre el bosque primario y los otros tipos de cobertura.

Descriptores: Agrosilvicultura, manejo del suelo, área degradada.

\section{INTRODUÇÃO}

O modelo tradicional de exploração das atividades agropecuárias na Amazônia caracteriza-se pela ocupação desordenada do espaço territorial, por meio de práticas relacionadas ao desmatamento e queimadas que promoveram modificações extensas em florestas nativas da região, nas últimas décadas, além disso, em razão do manejo inadequado, muitos solos tornaram-se improdutivos (MASCARENHAS, 2017).

Portanto, uma alternativa para manter ou melhorar a fertilidade do solo é a adoção de sistemas de uso da terra que permitam garantir o acúmulo de material orgânico, de modo que se proporcione um balanço entre a adição e perdas de nutrientes, haja vista que os estoques de matéria orgânica em qualquer agroecossistema são obtidos pela interação dos fatores que determinam sua formação e àqueles que promovem sua decomposição (CAMPOS et al., 2013).
Desta maneira, os sistemas agroflorestais (SAF) enquadram-se neste contexto, pois são baseados em cultivos que favorecem a ciclagem de nutrientes, a partir da associação dos extratos aéreos e subterrâneos entre espécies florestais e agrícolas, em diversos arranjos temporais e espaciais com pouco ou nenhum revolvimento do solo, os quais representam vantagens sobre os sistemas de cultivo convencionais em relação a manutenção da fertilidade do solo (LENCI et al., 2018).

Dentre as vantagens, pode-se destacar a promoção de microclima mais favorável aos processos biológicos que afetam a decomposição da biomassa; melhoria na estrutura do solo e proteção contra a erosão; conservação da água no lençol freático; diversificação da microbiota e mesofauna benéficas aos solo; menor proliferação de pragas e doenças; menor incidência de plantas daninhas; conservação da biodiversidade (fauna e flora); proteção da área contra queimadas e eficiente ciclagem de nutrientes (SILVEIRA et al., 2007). 
Sendo assim, dentre os arranjos de SAF que podem apresentar os benefícios mencionados, destacam-se dois modelos: multiestratificado, caracterizado pelo cultivo de espécies florestais e agrícolas perenes de grande, médio e pequeno porte; e o de capoeira manejada, cujas características são semelhantes às condições de uma floresta natural, pois consistem na melhoria da capoeira tradicional, por meio do enriquecimento e intervenções (desbaste e corte de cipós) visando auxiliar a regeneração natural, reestabelecer a fertilidade do solo e antecipar e recompor o potencial produtivo do ecossistema, para permitir a continuidade do ciclo de utilização do solo (REGO e KATO, 2017).

Nessa perspectiva, objetivou-se com este trabalho avaliar as condições químicas do solo com diferentes sistemas agroflorestais no município de Ouro Preto do Oeste, Rondônia, localizado na Amazônia Sul-Ocidental.

\section{MATERIAIS E MÉTODOS}

\section{Caracterização da área de estudo}

O presente estudo foi realizado com base em um experimento implantado no ano de 1996, na Estação Experimental Ouro Preto - ESEOP, pertencente à Comissão Executiva do Plano da Lavoura Cacaueira (CEPLAC), localizada em Ouro Preto do Oeste, Rondônia (Figura 1), idealizado para avaliação de modelos de sistemas agroflorestais visando propiciar alternativas aos produtores rurais para recuperação de áreas degradadas no estado de Rondônia.
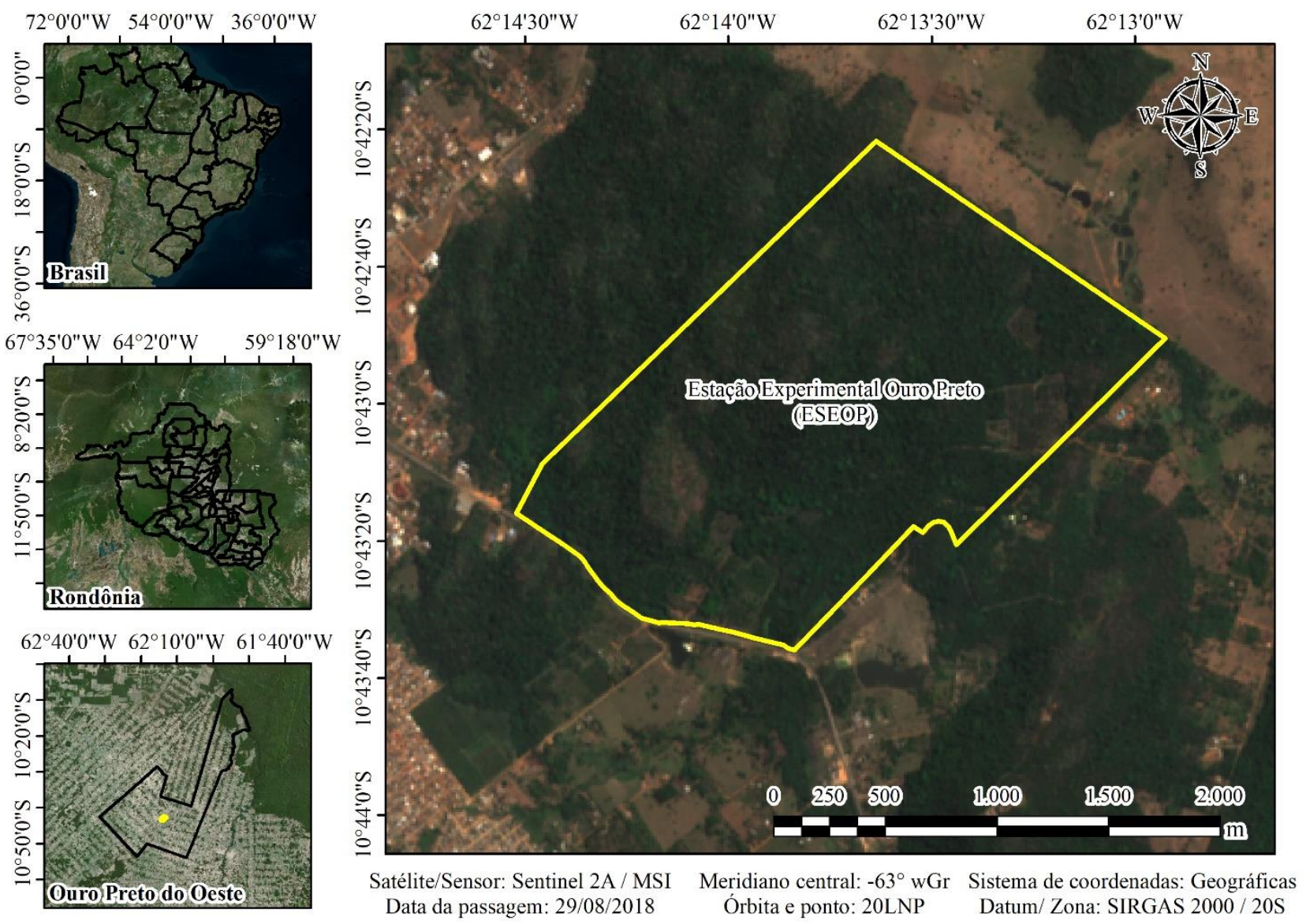

Figura 1 - Localização da área de estudo no município de Ouro Preto do Oeste, Rondônia. 
O clima da região, conforme a classificação de Köppen-Geiger, é do tipo Am (monções), quente e úmido, com precipitação anual em torno de 2000 $\mathrm{mm}$, sendo que o período chuvoso se concentra entre os meses de novembro e abril. A temperatura média mensal é de $25{ }^{\circ} \mathrm{C}$ e a umidade relativa do ar média mensal é em torno de $80 \%$ (ALVARES et al., 2013). O solo da região é classificado como Argissolo Vermelho-Amarelo Eutrófico (PVAe) (SANTOS et al., 2018).

\section{Caracterização dos tratamentos}

$\mathrm{O}$ experimento foi instalado em área de capoeira, onde foram avaliados três sistemas de cultivo, sendo eles:

Tratamento 1 (T1): Sistema agroflorestal com cacaueiros clonais e fruteiras perenes. $\mathrm{Na}$ implantação foi realizada a limpeza da área, seguida do plantio de cacaueiros sombreados com espécies frutíferas perenes, como fruta-pão, cupuaçu, abacateiro, mangueira, em parcela útil de $18 \mathrm{~m}$ x 30m. As fruteiras foram dispostas de forma aleatória. Os cacaueiros foram implantados após dois anos e meio, quando as fruteiras apresentavam sombra para este cultivo. $\mathrm{O}$ espaçamento adotado para o cacaueiro foi de $2 \mathrm{~m} \times 1,5 \mathrm{~m}$.

Tratamento 2 (T2): Trilhamento na capoeira com abertura de faixas (roçagem, encoivaramento) e plantio de essências florestais, como freijó-louro, bandarra, andiroba, ipê, itaúba e breu. Após a abertura das faixas $(3 \mathrm{~m} \times 30 \mathrm{~m}$ de comprimento, no sentido Leste-Oeste) foram plantadas mudas de essências florestais no espaçamento de $6 \mathrm{~m} \times 2 \mathrm{~m}$. A parcela total possui três fileiras (situadas no centro das faixas) com 16 plantas cada, metade para cada uma das seis espécies florestais selecionadas, com o objetivo de facilitar os trabalhos na época de corte. Neste tratamento, a área aberta com as faixas representou $50 \%$ da área total da parcela, enquanto a área remanescente permaneceu como capoeira no processo de regeneração natural.

Tratamento 3 (T3): Capoeira enriquecida com plantio de essências florestais, como cedro-rosa, castanheira do Brasil, bandarra e andiroba. Para o plantio e manutenção das mudas deste sistema foi feito apenas a abertura das covas dentro da capoeira, e, quando necessário, o coroamento até a emergência das plantas.

Tratamento 4 (T4): Floresta primária em área adjacente à área experimental, com parcelas de $18 \mathrm{~m} \mathrm{x}$ $30 \mathrm{~m}$, semelhante aos demais tratamentos.

\section{Coleta e análise do solo}

As coletas das amostras de solo foram realizadas por meio do uso de uma sonda metálica, retirando-se seis amostras compostas por unidade amostral de cada sistema agroflorestal, nas profundidades de $0 \mathrm{~cm}$ a $20 \mathrm{~cm}$ e $20 \mathrm{~cm}$ a $40 \mathrm{~cm}$. O período de coleta foi no mês de outubro de 2012, considerado final do período seco e início do período chuvoso na região.

As amostras de solo foram encaminhadas ao Laboratório de Solos da Embrapa Rondônia, no qual foram realizadas as determinações de $\mathrm{pH}$ em água, dos teores de $\mathrm{Al}, \mathrm{P}, \mathrm{K}, \mathrm{Ca}$ e $\mathrm{Mg}$ disponíveis e da matéria orgânica do solo, com base na metodologia preconizada (CLAESSEN, 1997). Além disso, foram calculados os parâmetros de fertilidade do solo, como, soma de bases (SB), CTC efetiva (t) e saturação por bases $(\mathrm{V} \%)$.

\section{Delineamento experimental e análise dos resultados}

$\mathrm{O}$ delineamento experimental adotado foi em blocos casualizados, com três repetições por sistema agroflorestal, conforme as premissas indicadas por Costa Junior (2008) e Mascarenhas et al. (2017), pois as áreas estavam nas mesmas condições 
edafoclimáticas. Os dados obtidos foram submetidos à análise de variância e a comparação de médias foi realizada por meio da aplicação do teste de ScottKnott $(\mathrm{p}<0,05)$, utilizando-se o pacote estatístico SISVAR 5.6 (FERREIRA, 2014).

\section{RESULTADOS E DISCUSSÃO}

Entre os SAF avaliados, para os teores de cálcio, alumínio e soma de bases, nas profundidades de $0 \mathrm{~cm}$ a $20 \mathrm{~cm}$ e $20 \mathrm{~cm}$ a $40 \mathrm{~cm}$ não foram observadas diferenças, enquanto que os teores de potássio, magnésio e CTC efetiva diferiram apenas na profundidade de $20 \mathrm{~cm}$ a $40 \mathrm{~cm}$ (Tabela 1), indicando que ao menos uma das coberturas pode promover variações nas características químicas do solo estudado.

Tabela 1. Resumo das análises de variância, coeficiente de variação e médias gerais para os nutrientes $\mathrm{P}, \mathrm{K}, \mathrm{Ca}$, $\mathrm{Mg}, \mathrm{pH}$, teor de Al, matéria orgânica (MO), soma de bases (SB), CTC efetiva (t) e saturação de bases (V) obtidos de análises de solos de diferentes tipos de uso agroflorestal em Ouro Preto do Oeste, Rondônia, Brasil.

\begin{tabular}{|c|c|c|c|c|c|c|c|c|c|c|c|}
\hline \multicolumn{12}{|c|}{ Quadrado Médio } \\
\hline \multirow{2}{*}{$\mathrm{FV}$} & \multirow{2}{*}{ GL } & \multirow{2}{*}{$\begin{array}{c}\mathrm{pH} \text { em } \\
\text { água }\end{array}$} & $\mathrm{P}$ & $\mathrm{K}$ & $\mathrm{Ca}$ & $\mathrm{Mg}$ & $\mathrm{Al}$ & SB & $\mathrm{t}$ & \multirow{2}{*}{$\begin{array}{c}\mathrm{MO} \\
\left(\mathrm{g} \mathrm{kg}^{-1}\right)\end{array}$} & \multirow{2}{*}{$\begin{array}{c}\mathrm{V} \\
(\%)\end{array}$} \\
\hline & & & \multicolumn{4}{|c|}{$\left(\mathrm{mg} \mathrm{dm}^{-3}\right)$} & \multicolumn{3}{|c|}{$\left(\mathrm{mmol}_{\mathrm{c}} \mathrm{dm}^{-3}\right)$} & & \\
\hline \multicolumn{12}{|c|}{$0 \mathrm{~cm} \mathrm{a} 20 \mathrm{~cm}$} \\
\hline Trat. & 3 & $0,08^{\mathrm{ns}}$ & $0,01^{\mathrm{ns}}$ & $1,22^{\mathrm{ns}}$ & $63,64^{*}$ & $3,66^{\mathrm{ns}}$ & $3,15^{*}$ & $95,66^{*}$ & $85,47^{\mathrm{ns}}$ & $21,1^{\mathrm{ns}}$ & $83,33^{\text {ns }}$ \\
\hline Erro & 6 & 0,01 & 0,25 & 0,31 & 9,60 & 2,38 & 0,41 & 13,93 & 10,57 & 7,72 & 233,08 \\
\hline $\mathrm{CV}(\%)$ & & 2,33 & 25,0 & 28,13 & 18,28 & 31,90 & 79,29 & 15,72 & 13,24 & 23,73 & 30,53 \\
\hline Média & & 5,58 & 2,00 & 1,98 & 16,95 & 4,84 & 0,80 & 23,74 & 24,55 & 11,71 & 50,00 \\
\hline \multicolumn{12}{|c|}{$20 \mathrm{~cm} \mathrm{a} 40 \mathrm{~cm}$} \\
\hline Trat. & 3 & $1,7^{\mathrm{ns}}$ & $8,84^{\mathrm{ns}}$ & $3,25^{*}$ & $66,77^{*}$ & $10,34^{*}$ & $7,29^{*}$ & $117,49^{*}$ & $296,57^{*}$ & $32,81^{\mathrm{ns}}$ & $98,44^{\mathrm{ns}}$ \\
\hline Erro & 6 & 1,18 & 8,84 & 0,38 & 11,49 & 1,89 & 0,60 & 12,78 & 56,58 & 4,09 & 82,02 \\
\hline $\mathrm{CV}(\%)$ & & 21,30 & 160,0 & 33,92 & 30,38 & 36,82 & 49,52 & 21,42 & 16,83 & 25,77 & 19,55 \\
\hline Média & & 5,10 & 1,0 & 1,82 & 11,16 & 3,73 & 1,57 & 16,68 & 18,25 & 7,85 & 46,33 \\
\hline
\end{tabular}

Em que: ${ }^{*}=$ Significativo a $5 \%$ de probabilidade $\mathrm{e}^{\mathrm{ns}}=$ não-significativo.

Contudo, para maior detalhamento destas diferenças, na Tabela 2 é possível observar quais sistemas promoveram influência características químicas do solo, dessa forma, os maiores teores de Al foram obtidos nos tratamentos $\mathrm{T} 1$ e $\mathrm{T} 4$, variando de $1,10 \mathrm{mmol}_{\mathrm{c}} \quad \mathrm{dm}^{3}$ a $2,13 \mathrm{mmolc} \quad \mathrm{dm}^{3}$, na profundidade de $0 \mathrm{~cm}$ a $20 \mathrm{~cm}$, e de $2,03 \mathrm{mmolc} \mathrm{dm}^{3}$ a $3,53 \mathrm{mmolc} \mathrm{dm}^{3}$, na profundidade de $20 \mathrm{~cm}$ a $40 \mathrm{~cm}$.

$\mathrm{Na}$ análise dos valores de $\mathrm{pH}$ observados nas duas profundidades, verificou-se que não houveram diferenças entre os tratamentos e os valores encontrados caracterizam um solo com acidez de nível médio, segundo Furtini Neto et al. (2001).

Provavelmente, estes resultados sejam provenientes do efeito positivo da mineralização da matéria orgânica no solo, que auxilia no incremento da capacidade de troca catiônica (CTC) e soma de bases, causando assim, a maior liberação de cátions e ânions trocáveis que são adsorvidos pelos colóides do solo.

A explicação deste comportamento foi abordada por Da Ros et al. (2017), ao mencionarem que os minerais secundários e as frações matéria orgânica apresentam-se, normalmente, como colóides e são os principais responsáveis pela atividade química dos solos.

Em complemento, Souza (2009) mencionou que, devido a essas características são formadas cargas elétricas na superfície dos colóides, permitindo com que a fase sólida retenha cátions e ânions liberados para a solução do solo pelo intemperismo dos minerais primários e decomposição da matéria 
orgânica ou, ainda adicionados na solução pela adubação.

Cidin et al. (2009), obtiveram resultados com cacaueiros e coqueiros em Ji-Paraná - RO, exceto para os teores de alumínio que foram mais baixos.

semelhantes em SAF com diferentes arranjos entre

Tabela 2. Valores médios do $\mathrm{pH}$, teor de $\mathrm{Al}$, matéria orgânica (MO), saturação de bases (V) e dos nutrientes $\mathrm{P}, \mathrm{K}$, $\mathrm{Ca}, \mathrm{Mg}$ obtidos em análises de solos nos diferentes tipos de uso agroflorestal em Ouro Preto do Oeste, Rondônia, Brasil.

\begin{tabular}{|c|c|c|c|c|c|c|c|c|c|c|}
\hline \multirow{2}{*}{ Tratamentos } & \multirow{2}{*}{$\begin{array}{l}\mathrm{pH} \text { em } \\
\text { água }\end{array}$} & \multirow{2}{*}{$\begin{array}{c}\mathrm{P} \\
\left(\mathrm{mg} \mathrm{dm}^{-3}\right)\end{array}$} & $\mathrm{K}$ & $\mathrm{Ca}$ & $\mathrm{Mg}$ & $\mathrm{Al}$ & SB & $\mathrm{t}$ & \multirow{2}{*}{$\begin{array}{c}\mathrm{MO} \\
\left(\mathrm{g} \mathrm{kg}^{-1}\right)\end{array}$} & \multirow{2}{*}{$\begin{array}{c}\mathrm{V} \\
(\%)\end{array}$} \\
\hline & & & \multicolumn{6}{|c|}{$\left(\mathrm{mmol}_{\mathrm{c}} \mathrm{dm}^{-3}\right)$} & & \\
\hline \multicolumn{11}{|c|}{$0 \mathrm{~cm}$ a $20 \mathrm{~cm}$} \\
\hline T1 & 5,56 & 2 & 1,14 & $13,47 b$ & 3,37 & $1,10 \mathrm{a}$ & $17,98 b$ & 19,08 & 8,20 & 52,33 \\
\hline $\mathrm{T} 2$ & 5,56 & 2 & 2,20 & $15,13 b$ & 4,63 & $0,00 \mathrm{~b}$ & $21,96 b$ & 21,96 & 11,23 & 45,33 \\
\hline T3 & 5,8 & 2 & 1,91 & $23,73 a$ & 5,80 & $0,00 \mathrm{~b}$ & $31,44 \mathrm{a}$ & 31,44 & 14,27 & 56,33 \\
\hline $\mathrm{T} 4$ & 5,4 & 2 & 2,66 & $15,47 b$ & 5,57 & $2,13 a$ & $23,70 b$ & 25,83 & 13,13 & 46,00 \\
\hline Média & 5,58 & 2 & 1,98 & 16,95 & 4,84 & 0,80 & 23,74 & 24,57 & 11,71 & 50,00 \\
\hline $\mathrm{CV}(\%)$ & 2,33 & 25 & 28,13 & 18,28 & 31,90 & 79,29 & 15,72 & 13,24 & 23,73 & 30,53 \\
\hline \multicolumn{11}{|c|}{$20 \mathrm{~cm} \mathrm{a} 40 \mathrm{~cm}$} \\
\hline $\mathrm{T} 1$ & 5,37 & 1 & $0,78 \mathrm{a}$ & $7,93 \mathrm{~b}$ & $1,80 \mathrm{~b}$ & $2,03 b$ & $10,51 b$ & $12,54 b$ & 4,63 & 41,67 \\
\hline $\mathrm{T} 2$ & 5,43 & 1 & $1,35 \mathrm{a}$ & $10,10 \mathrm{~b}$ & $2,50 \mathrm{~b}$ & $0,70 \mathrm{a}$ & $13,95 b$ & $14,65 b$ & 6,13 & 44,00 \\
\hline $\mathrm{T} 3$ & 5,63 & 1 & $1,93 a$ & $18,10 \mathrm{a}$ & $5,13 \mathrm{a}$ & $0,00 \mathrm{a}$ & $25,16 a$ & $25,16 a$ & 12,23 & 54,67 \\
\hline $\mathrm{T} 4$ & 5,21 & 1 & $3,22 b$ & $8,50 \mathrm{~b}$ & $5,50 \mathrm{a}$ & $3,53 b$ & $17,22 b$ & $20,75 a$ & 8,40 & 46,33 \\
\hline Média & 5,41 & 1 & 1,82 & 11,16 & 3,73 & 1,57 & 16,68 & 18,25 & 7,85 & 46,33 \\
\hline $\mathrm{CV}(\%)$ & 21,3 & 160 & 33,92 & 30,38 & 36,82 & 49,52 & 21,42 & 16,83 & 25,77 & 19,55 \\
\hline
\end{tabular}

Em que: T1= Cacau x Fruteiras; T2 = Trilha na capoeira; T3 = Capoeira; T4 = Floresta. Valores precedidos da mesma letra na coluna não diferem, significativamente, a 5\% de probabilidade pelo teste de Scott-Knott.

Os teores de $\mathrm{P}$ disponível foram considerados baixos nas duas profundidades para todos os sistemas analisados, com maior concentração na profundidade de $0 \mathrm{~cm}$ a $20 \mathrm{~cm}$, em função da sua baixa mobilidade no solo (DALCHIAVON et al., 2017), porém não houveram diferenças entre os tratamentos, à semelhança dos resultados encontrados por Cidin et al. (2009) em sistemas agroflorestais com cacaueiros e coqueiros.

Os estudos realizados por McGrath et al. (2000) em SAF com pupunheiras e cupuaçuzeiros, com seis anos de idade, auxiliam no entendimento deste resultado, pois os autores explicaram que sistemas agroflorestais com grande densidade de plantas e pouca incidência luminosa resultam na decomposição mais lenta da serrapilheira acumulada que por sua vez desacelera a mineralização de $\mathrm{P}$, devido a isso é possível que ocorra a redução na disponibilidade de $\mathrm{P}$, comprometendo a capacidade produtiva do sistema.

Ainda nesse contexto, Corrêa et al. (2006) ao avaliarem um sistema agroflorestal multiestratificado com espécies frutíferas e florestais em Rondônia, com espaçamentos maiores, também observaram maior estoque de $\mathrm{P}$ na profundidade de $0 \mathrm{~cm}$ a $20 \mathrm{~cm}$.

Em relação aos teores de $\mathrm{K}$, não foram verificados efeitos promovidos pelos diferentes SAF, sendo que na maioria dos tratamentos os teores encontrados são considerados médios $\left(1,35 \mathrm{mmol}_{\mathrm{c}}\right.$ $\mathrm{dm}^{3}$ a $3,22 \mathrm{mmol}_{\mathrm{c}} \mathrm{dm}^{3}$ ), a exceção para os teores no $\mathrm{T} 1$, considerados baixos $\left(1,14 \mathrm{e} 0,78 \mathrm{mmol}_{\mathrm{c}} \mathrm{dm}^{3}\right)$ nas profundidades de $0 \mathrm{~cm}$ a $20 \mathrm{~cm}$ e $20 \mathrm{~cm}$ a $40 \mathrm{~cm}$, respectivamente.

De forma semelhante, Ayres e Alfaia (2007), avaliando os efeitos da calagem e da adubação potássica em SAF no Projeto RECA em Rondônia, 
observaram níveis críticos deste nutriente nos solos, evidenciando sua escassez e a importância de sua reposição no sistema.

Da mesma forma, Corrêa et al. (2006), obtiveram maiores teores de $\mathrm{K}$ na associação de cacaueiros sombreados com gliricídia, nas profundidades de $0 \mathrm{~cm}$ a $20 \mathrm{~cm}$ e $20 \mathrm{~cm}$ a $40 \mathrm{~cm}$, isto demonstra a influência não apenas dos arranjos espaciais, mas também da composição florística dos SAF, pois algumas espécies serão mais eficientes na ciclagem de nutrientes em função do regime de desfolha e das características da biomassa produzida.

Quanto aos teores de Ca trocável, nos diferentes agroecossistemas estudados, houveram variações substanciais, contudo os valores são considerados médios na camada de $0 \mathrm{~cm}$ a $20 \mathrm{~cm}$ e baixos na camada de $20 \mathrm{~cm}$ a $40 \mathrm{~cm}$ de profundidade, independentemente do SAF analisado. No entanto, os maiores teores observados foram no tratamento T3 (capoeira), com 23,73 $\mathrm{mmol}_{\mathrm{c}} \mathrm{dm}^{3}$, na profundidade de $0-20 \mathrm{~cm}$, e de $18,10 \mathrm{mmol}_{\mathrm{c}} \mathrm{dm}^{3}$ na profundidade de $20-40 \mathrm{~cm}$.

Em complemento a esses aspectos, Ferreira $e t$ al. (2006) observaram que em ambiente equilibrado, onde não há a retirada da vegetação (mata), os teores de Ca tendem a serem superiores, e neste estudo, os maiores teores de Ca situaram-se na profundidade de $0 \mathrm{~cm}$ a $20 \mathrm{~cm}$, na qual foram registrados, também, os maiores teores de matéria orgânica. Segundo Leite (1987), este fato decorre de a maioria do Ca disponível provir da liteira que se decompõe e se incorpora ao solo através dos processos de lixiviação e deposição.

Para os teores de $\mathrm{Mg}$, os valores obtidos são considerados de baixos a médios (MALAVOLTA et al., 2002), além disso, variações significativas foram observadas apenas para a profundidade de $20 \mathrm{~cm}$ a $40 \mathrm{~cm}$, destacando-se os tratamentos $\mathrm{T} 4$, com o maior teor $\left(5,50\right.$ mmolc $\left.\mathrm{dm}^{3}\right)$, e $\mathrm{T} 1$ com o menor $(1,80$ mmolc $\left.\mathrm{dm}^{3}\right)$.

Além disso, as pesquisas de Wandelli et al. (2002) e Cidin et al. (2009), auxiliam na elucidação destes resultados, pois os autores explicaram que há redução do teor de $\mathrm{Mg}$ ao passo que se aumenta a profundidade do solo, o que indica a importância da matéria orgânica e da ciclagem de nutriente no teor desse elemento no solo.

Ainda, Menezes et al. (2008) comparando solos sob sistemas agroflorestais e floresta em Rondônia, observaram que os valores de $\mathrm{pH}, \mathrm{Ca}$ e $\mathrm{Mg}$ foram sempre maiores nos sistemas agroflorestais do que em solos de florestas remanescentes, o que é atribuído ao aporte de nutrientes que restaram da queima anterior da floresta, ocorrida entre um e oito anos antes da instalação do sistema agroflorestal.

Para a variável soma de bases (SB), o valor obtido pode ser classificado como médio $(23,74$ mmolc $\mathrm{dm}^{3}$ ) na profundidade de $0 \mathrm{~cm}$ a $20 \mathrm{~cm}$, com exceção para o $\mathrm{T} 1$ (17,98 mmolc $\left.\mathrm{dm}^{3}\right)$ que é considerado baixo. Por outro lado, os maiores valores encontrados foram para o tratamento $\mathrm{T} 3,31,44$ mmolc $\mathrm{dm}^{3}$ e 25,16 mmolc $\mathrm{dm}^{3}$, nas profundidades de $0 \mathrm{~cm}$ a $20 \mathrm{~cm}$ e $20 \mathrm{~cm}$ a $40 \mathrm{~cm}$, respectivamente, em consequência dos teores de $\mathrm{Ca}$ e $\mathrm{Mg}$ nessas profundidades.

Na CTC efetiva (t), que é a quantidade de cátions trocáveis do solo, nas condições da amostra, não houveram diferenças na profundidade de $0 \mathrm{~cm}$ a $20 \mathrm{~cm}$, com valor médio de $24,55 \%$, porém detectouse variações na profundidade de $20 \mathrm{~cm}$ a $40 \mathrm{~cm}$, decorrente da diferença na SB (Ca e Mg) (CIDIN et al., 2009).

Para os teores de matéria orgânica (MO), não se observou diferença significativa nas duas profundidades analisadas, à semelhança dos resultados obtidos por McGrath et al. (2000) e Corrêa et al. (2006). Ainda nesse contexto, as pesquisas de 
Araújo e Collier (2005) com SAF, lavoura e mata, evidenciaram que não houve diferença significativa entre os sistemas pesquisados, na profundidade de $0 \mathrm{~cm}$ a $40 \mathrm{~cm}$, verificando-se que no período seco, maiores valores de MO na mata.

A saturação de bases (V\%), que indica a proporção desta na CTC do solo, apresentou valores médios (entre 40,1 e $60,0 \%$ ) em todos os tratamentos e sem diferenças significativas entre eles. No entanto, Cidin et al. (2009) obtiveram baixa saturação por bases em diferentes arranjos com cacaueiros e coqueiros, atribuindo isso a provável lenta decomposição do material existente na serapilheira.

Adicionalmente, nos resultados obtidos por Iwata et al. (2012), ao avaliarem efeitos dos sistemas agroflorestais nos atributos químicos em Argissolo Vermelho-Amarelo, foram observados valores de CTM semelhantes aos encontrados neste trabalho.

\section{CONCLUSÃO}

O cálcio e o alumínio foram os nutrientes que mais variaram nos diferentes tipos de cobertura e profundidades do solo e para $\mathrm{pH}$, teores de fósforo e matéria orgânica não foram observadas diferenças entre a floresta e os demais tratamentos.

Os teores de matéria orgânica e P no solo não diferiram entre as coberturas vegetais avaliadas, o que indica que os modelos agroflorestais conservam algumas propriedades químicas do solo com valores similares ao da floresta natural durante o período considerado.

Todos os autores declararam não haver qualquer potencial conflito de interesses referente a este artigo.

\section{REFERÊNCIAS}

ALVARES, C.A.; STAPE, J.L. SENTELHAS, P.C. GONÇALVES, J.L.M.; SPAROVEK, G. Köppen's climate classification map for Brazil. Meteorologische Zeitschrift, Stuttgart, v.22, n.6, p.711-728, 2013.
ARAÚJO, G.P.; COLLIER, L. Parâmetros de fertilidade dos solos em sistemas agroflorestais em Esperantina - TO. In: $6^{\circ}$ CONGRESSO BRASILEIRO DE SISTEMAS AGROFLORESTAIS, 2005, Salvador, BA. Sociedade Brasileira de Sistemas Agroflorestais. Salvador: SBSAF, 2005.

AYRES, M.I.C.; ALFAIA, S.S. Calagem e adubação potássica na produção do cupuaçuzeiro em sistemas agroflorestais da Amazônia Ocidental. Pesquisa Agropecuária Brasileira, Brasília, v.42, n.7, p.957963, mar. 2007.

CAMPOS, L.P.; LEITE, L.F.C.; MACIEL, G.A.; BRASIL, E.L.; IWATA, B.F. Estoques e frações de carbono orgânico em Latossolo Amarelo submetido a diferentes sistemas de manejo. Pesquisa Agropecuária Brasileira, Brasília, v.48, n.3, p.304312, mar. 2013.

CIDIN, A.C.M.; CORREAA, F.L.O.; PEQUENO, P.L.L.; ALMEIDA, C.M.V.C.; MÜLLER, M.W.; MACEDO, R.G.; GAMA-RODRIGUES, A.C. Fertilidade do solo em sistema agroflorestal com cacaueiros e coqueiros em Ji-Paraná, Rondônia, Brasil. Agrotrópica, Ilhéus, v.21, n.1, p.67-72. jan. 2009.

CLAESSEN, M.E.C. Manual de métodos de análise de solos. 2. ed. Rio de Janeiro: Embrapa-CNPS, 1997. $212 \mathrm{p}$.

CORRÊA, F.L.O.; RAMOS, J.D.; GAMARODRIGUES, A.C.; MÜLLER, M.W.; MACEDO, R.G.; SOUZA, C.A.S.; ALVARENGA, M.I.N. Ciclagem de nutrientes em sistema agroflorestal com espécies frutíferas e florestais em Rondônia, Brasil. Agrotrópica, v.18, n.1, p.71-82, nov. 2006.

COSTA JUNIOR, C. Estoque de carbono e nitrogênio e agregação do solo sob diferentes sistemas de manejo agrícola. 2008. $140 \mathrm{f}$. Dissertação (Mestrado em Ciências) - Universidade de São Paulo, Piracicaba, 2008.

DA ROS, C.O.; SILVA, V.R.; SILVESTRIN, T.B.; SILVA, R.F.; PESSOTTO, P.P. Disponibilidade de nutrientes e acidez do solo após aplicações sucessivas de água residuária de suinocultura. Revista Brasileira de Tecnologia Agropecuária, v.1, n.1, p. 35-44, jul. 2017.

DALCHIAVON, F.C.; DAL BEM, E.A.; CARVALHO, M.P.; SARTO, M.D.; MONTANARI, R.; KANEKO, F.H. Relações da produtividade de cana-de-açúcar com atributos químicos de um 
Argissolo. Revista de Ciências Agrárias, Lisboa, v.40, n.4, p.756-769, jan. 2017.

FERREIRA, D. F. Sisvar: a Guide for its Bootstrap procedures in multiple comparisons. Ciência e Agrotecnologia, Lavras, v.38, n.2, p.109-112, set./out. 2014.

FERREIRA, S.J.F.; LUIZÃO, F.J.; MIRANDA, S.A.; SILVA, M.S.R.; VITAL, A.R.T. Nutrientes na solução do solo em floresta de terra firme na Amazônia Central submetida à extração seletiva de madeira. Acta Amazonica, Manaus, v.36, n.1, p.5968, jan./mar. 2006.

FURTINI NETO, A.E.; VALE, F.R.; RESENDE, A.V.; GUILHERME, L.R.G.; GUEDES, G.A.A. Fertilidade do Solo. Lavras: UFLA, 2001. 252p.

IWATA， B.F.; LEITE， L.F.; ARAÚJO, A.S.F.; NUNES, L.A.P.L.; GEHRING, C.; CAMPOS, L.P. Sistemas agroflorestais e seus efeitos sobre os atributos químicos em Argissolo Vermelho-Amarelo do Cerrado piauiense. Revista Brasileira de Engenharia Agrícola e Ambiental, v.16, n.7, p. 730-738, maio. 2012.

LEITE, J.O. Característica do manto detrítico e sua relação com a fertilidade da camada superficial de solo em plantações de cacau. Revista Brasileira de Ciência do Solo, Campinas, v.11, p.45-49, jan./abr. 1987.

LENCI, L.H.V.; SOUZA, E.F.M; MASCARENHAS, A.R.P; TSUKAMOTO FILHO, A.A.; SOARES, G.S. Aspectos fitossociológicos e indicadores da qualidade do solo em sistemas agroflorestais. Nativa, Sinop, v.6, n. especial, p.745-753, dez. 2018.

MALAVOLTA, E.; PIMENTEL-GOMES, F.; ALCARDE, J.C. Adubos e adubações. São Paulo: Nobel. 2002. 200p.

MASCARENHAS, A.R.P.; SCOTTI, M.S.; MELO, R.R.; CORREAA， F.L.O.; SOUZA， E.F.M.; ANDRADE, R.A.; BERGAMIN, A.C.; MÜLLER, M.W. Atributos físicos e estoques de carbono do solo sob diferentes usos da terra em Rondônia, Amazônia Sul-Ocidental. Pesquisa Florestal Brasileira, Colombo, v.37, n.89, p.19-27, jan./mar. 2017.

McGRATH, D.A.; COMERFORD, N.B.; DURYEA, M.L. Litter dynamics and monthly fluctuation in soil phosphorus availability in Amazonian agroforest. Forest Ecology Management, Flagstaff, v.131, n.1, p.167-181, jun. 2000.
MENEZES, J.M.T.; LEEUWEN, J.; VALERI, S.V.; CRUZ, M.C.P.; LEANDRO, R.C. Comparação entre solos sob uso agroflorestal e em florestas remanescentes adjacentes, no norte de Rondônia. Revista Brasileira de Ciência do Solo, Viçosa, v.32, n.2, mar./abr. 2008.

REGO, A.K.C.; KATO, O.R. Agricultura de corte e queima e alternativas agroecológicas na Amazônia. Novos Cadernos NAEA, Belém, v.20, n.3, p.203224, set./dez. 2017.

SANTOS, H. G. dos; JACOMINE, P. K. T.; ANJOS, L. H. C. dos; OLIVEIRA, V. A. de; LUMBRERAS, J. F.; COELHO, M. R.; ALMEIDA, J. A. de; ARAUJO FILHO, J. C. de; OLIVEIRA, J. B. de; CUNHA, T. J. F.. Sistema brasileiro de classificação de solos. 5. ed. Brasília: Embrapa, 2018. $356 \mathrm{p}$.

SOUZA F.R. Efeito do manejo do solo e do gesso residual na cultura da soja em atributos físicos de um Latossolo vermelho distroférrico. 2009. $55 \mathrm{f}$. Dissertação (Mestrado em Produção Vegetal) Universidade Federal da Grande Dourados, Dourados, 2009.

SILVEIRA, N.D.; PEREIRA, M.G.; POLIDORO, J.C.; TAVARES, S.R.L.; MELLO, R.B. Aporte de nutrientes e biomassa via serrapilheira em sistemas agroflorestais em Paraty (RJ). Ciência Florestal, Santa Maria, v.17, n.2, p.129-136, abr./jun. 2007.

WANDELLI, E.V.; FERREIRA, F.; SOUSA, G.F.; SOUSA, S.G.A. de; FERNANDES, E.K.M. Exportação de nutrientes de sistemas agroflorestais através da colheita: $\mathrm{O}$ valor dos resíduos dos frutos Amazônicos. In: $4^{\circ}$ Congresso Brasileiro de Sistemas Agroflorestais, 2002, Ilhéus, BA. Comissão Executiva do Plano da Lavoura Cacaueira. Ilhéus: CEPLAC, 2002. 